\title{
Self-stimulation behavior: Methods evaluating intracranial reward and their relations with motivational states
}

\author{
PH. DE WITTE and R. BRUYER \\ Laboratoire de Neurophysiologie, Université Catholique de Louvain, B-1200 Brussels, Belgium
}

\begin{abstract}
Monopolar electrodes were chronically implanted in the posterior lateral hypothalamus of male rats. Animals exhibiting steady self-stimulation behavior were allowed to self-administer brain stimulation using various procedures: a continuous reinforcement schedule, fixed ratio procedures, choice method, cost methods, consummatory procedures, determination of the extinction of self-stimulation behavior, self-regulation of train duration stimulation, and the switch-off methor. Results of factor analysis support the difficulty of objectively favoring one method over another. Three "motivational procedures," that is, feeding, sexual, and aggressive behaviors, were also designed to study the relationships between drive state and rewarding strength elicited by hypothalamic stimulations. Interpretations of these relationships in terms of drive reduction or drive induction hypotheses show how complex the nature of the relation is, indicating that these interpretations depend on the ability to interpret the multifactorial nature of the reinforcing effect.
\end{abstract}

Since Valenstein's (1964) description of the problems encountered in the measurement of rewarding brain stimulation, few behavioral publications have been able to precisely define valid methods for measuring the rewarding effect induced by brain stimulations. Moreover, variability in the results concerning the measure of rewarding effect may generally be attributed to variations in procedures. It seems important, therefore, to specify a criterion to provide an analysis of the discrepancies among results and to reduce differences between procedures.

In the case of the self-stimulation behavior, independent and dependent variables could be single or multiple. Actually, the estimation of the rewarding effect, that is, the dependent variable, has been made by employing only a single electrical parameter of brain stimulation (Olds \& Olds, 1963; White, 1975), thus permitting determination of the presence or absence of an effect of rewarding brain stimulation on various behaviors. Others (Keesey, 1964; Olds \& Milner, 1954) have used several electrical parameters of brain stimulation, allowing for a continuum of the independent variable, while using a single method to measure the rewarding effect. Also, reinforcement schedules have been used to study the

This article is dedicated to the memory of M. Beyra. The authors are indebted to professor M. Meulders for his constructive criticism, and they wish to thank Fr. Huybrechts-Ameels, L. Gomes da SilvaLemmens, Fr. Langhendries-Vrins, A. Mombaerts, M. Ch. Hendrick, J. Schouppe, and Ch. Stoquart for their technical assistance. The authors' address for correspondence is: Laboratoire de Neurophysiologie, Université Catholique de Louvain, U.C.L. 5449, Avenue Hippocrate, 54, B-1200 Bruxelles, Belgium. variability of the effect of brain stimulations (Brodie, Moreno, Malis, \& Boren, 1960; Gallistel, 1964; Hodos \& Valenstein, 1962; Sidman, Brady, Conrad, \& Schulman, 1955).

If one employed many different stimuli (i.e., a continuum in the independent variable) in different experimental procedures (i.e., many dependent variables), one could hopefully generate more valid criteria to estimate the value of each method. In a previous article, Beyra (1974) operationally defined reinforcement as a set of physiological variables that increased the probability of a response. He used five procedures to study the reinforcement elicited by brain stimulation and compared these methods through factor analysis. Our aim was to study a larger set of procedures and to compare methods quantifying the rewarding strength of brain stimulations.

Our hypothesis was the following: Variations in reward strength as they appear in the variety of results obtained through the use of different methods are due mainly to differences in the procedure used to estimate rewarding stimulations rather than to the rewarding value induced by the brain stimulation. Furthermore, it seems that the reward strength of hypothalamic stimulation is highly related to the internal drive state elicited (Beyra, 1976; De Witte, 1978). The relationship between the reinforcing state and the drive state elicited by hypothalamic stimulation is interpreted according to the general "mood" factor, the drive induction hypothesis, and the drive reduction hypothesis. Each of these interpretations seems to verify a link in the chain of the overall relationship. 


\section{METHOD}

\section{Subjects, Electrodes, and Histology}

The subjects were male albino rats of the Wistar strain, each weighing $300 \mathrm{~g}$ at the time of operation. Each rat was implanted with the monopolar nickel-chrome electrodes $(.255 \mathrm{~mm}$ in diameter), insulated except for the cross section of the tip. The region aimed at was the posterolateral hypothalamus. The indifferent electrode was placed $1 \mathrm{~mm}$ anterior to the bregma. The electrodes were stereotaxically implanted bilaterally, according to the following coordinates: A, $3.5 \mathrm{~mm}$ posterior to bregma; $\mathrm{L}, 1.2 \mathrm{~mm}$ from the midline; $\mathrm{H}, 8.3 \mathrm{~mm}$ below the skull surface.

After the operation, the animals were allowed to recover for 1 week before training for self-stimulation began. Pressing a lever produced a .5-sec train of negative rectangular pulses of .1 msec duration, delivered at a frequency of $100 \mathrm{~Hz}$. Current threshold for self-stimulation behavior ranged from 70 to $250 \mu \mathrm{A}$ and was monitored by means of an oscilloscope. Rats were submitted to daily training sessions until the barpressing rate became steady. Rats that did not exhibit self-stimulation behavior were eliminated.

Following termination of the experiment, the subjects were sacrificed. The brains were removed from the skull and put in Formalin solution for 10 days. The brains were then frozen, sectioned at $100 \mu$, and stained with cresyl-violet.

\section{Training and Apparatus}

One week after the operation, the animals were trained to selfstimulate in wooden boxes $(30 \mathrm{~cm}$ long, $12.5 \mathrm{~cm}$ deep, and $30 \mathrm{~cm}$ high), using a force of $30 \mathrm{~g}$ on the aforementioned lever to produce a stimulus. After 1 week of self-stimulation training, the barpressing rate became steady (150 barpresses per $5 \mathrm{~min}$ ) during 60-min daily sessions.

\section{Procedure}

\section{Measure of the Strength of the Reinforcing Effect}

Rats exhibiting steady self-stimulation behavior were distributed randomly in each experimental procedure. Sample groups of six rats were formed, using two criteria to obtain homogeneity. First, rats were selected on the basis of the self-stimulation level, that is, self-stimulation behavior that was similar from subject to subject during the training sessions. Second, they were chosen on the basis

Table 1 Parameters of the 38 Electrical Brain Stimuli



Note-Each intracranial stimulus is identified by a letter code (first column) and ranked according to the train duration (TD) in seconds, the pulse duration (PD) in milliseconds, and the frequency of pulses $(F)$ in hertz. The intensity remains constant at the self-stimulation threshold. of a histological analysis that showed that the electrode tips of the subjects were distributed randomly in the aimed at area. The animals received 38 combinations of the intracranial electrical brain stimuli; the train duration (TD) was varied from .03 to $5.3 \mathrm{sec}$, the pulse duration (PD) from .05 to $.2 \mathrm{msec}$, and the frequency of pulses (F) from 100 to $400 \mathrm{~Hz}$. Table 1 presents these combinations of electrical parameters. The 38 electrical combinations were presented to the rats according to a predesigned random order; each rat received the same order of stimulation presentation. For each experimental procedure, the combinations were administered five times for $5 \mathrm{~min}$ (i.e., $150 \mathrm{~min}$ per electrical combination for the sample of six rats). Twenty-eight experimental procedures were designed to quantify the rewarding effect of the 38 electrical combinations, and each was performed with a group of six rats. In a previous article, Beyra (1974) described 5 of the 28 procedures used in this study. The experimental procedures were the following.

Continuous reinforcement (CRF). A device similar to that used in self-stimulation training was used.

The absolute number of brain stimulations received by pressing a bar during one 5-min session for each of the 38 electrical combinations was obtained. The rats were exposed to two daily sessions of 30 min each (i.e., 12 combinations a day). At the end of each presentation, the number of stimulations received was recorded (Table 2).

Fixed ratio procedures (FR3, FR5, FR10, FR15). The apparatus was the same as that used in the CRF procedure.

Three, 5, 10, and 15 barpressings earned the rat one brain stimulation. The absolute number of brain stimulations during the 5 -min sessions was recorded (Table 2 ). The subjects were adapted to the FR schedules; in other words, the FR experiments began when rats performed the requirements of the FR adequately. It must be noted that the number of barpresses made while the brain was being stimulated was subtracted from the following FR requirements. For example, in the FR5 procedure, if an animal pressed the bar two times during a 4-sec train duration of brain stimulation, then three barpresses were required to produce the next brain stimulation.

Self-regulation method (SR). A device similar to that used in the CRF procedure was used.

The group of six rats was trained to regulate the duration of brain stimulation during a 15-min daily session for 1 week. Pushing down a lever turned on the brain stimulation; letting up on the lever switched it off. The pulse duration and the frequency were selected by the experimenter according to the following experimental procedure: Six electrical combinations were chosen-$.5-100, .1-100, .2-100, .2-200, .2-300$, and $.2-400 \mathrm{~Hz}$. The current intensity was held at the threshold at which the subject selfstimulated. Once the self-regulation behavior was acquired, each subject was exposed to five sessions of $10 \mathrm{~min}$ each for each electrical combination of frequency and pulse duration, two sessions in the morning and two sessions in the afternoon. Thirty minutes separated each session. Five hours' worth of performance with each of the six electrical combinations was then recorded. The frequency of barpressing (BPR), as described in Table 2, was recorded. We assumed that the more a particular electrical combination was selected, the more rewarding that electrical stimulus was.

Switch-off procedure (OFF). The device used was the same as that used in the CRF and FR procedures.

Brain stimulation was delivered automatically to the rat according to the self-stimulation rate established in the CRF procedure. Each barpress switched off a discontinuous brain stimulation for $1 \mathrm{sec}$. The number of switch-offs was recorded and subtracted from an arbitrary value (i.e., 15). These modified numbers are presented in Table 2. Our conclusion was that the less frequently a brain stimulus was switched off, the more rewarding it was (RW).

Consummatory procedures (CRFM, FR3M, FR5M, FR10M, FR15M, OFFM, and SRM; CRFT, FR3T, FR5T, FR10T, FR15T, OFFT, and SRT). This set of procedures concerns the amount of reward consumed during self-stimulation, self-regulation, and switch-off sessions. Even though a classical consummatory be- 


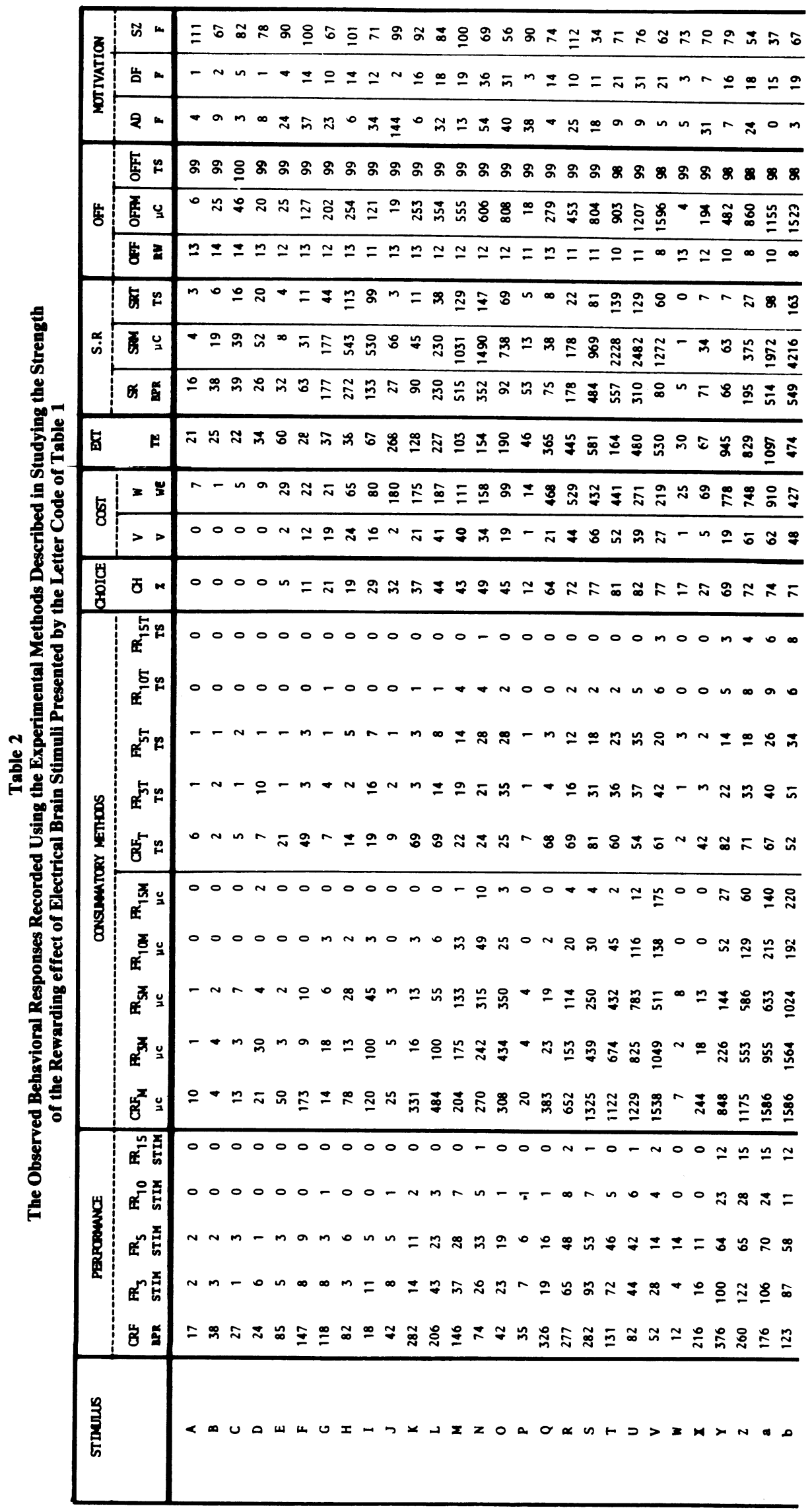




havior does not exist in self-stimulation, if one knows the amount of electrical charge $(Q=$ intensity $\times$ pulse duration $\times$ frequency) the animal receives and the amount of time during which the animal is stimulated (train duration), the amount of electricity consumed may be a measure of the strength of the reinforcer.

The amount of electrical charge received by the animals was calculated using the number of delivered stimulations in the CFR, FR, SR, and OFF methods, then multiplying first by $Q$ and then by the train duration. To express the obtained value in microcoulombs $(\mu \mathrm{C})$, the intensity was multiplied by the constant $10^{-3}$. This method determines the amount of electrical charge and the time the animal self-administered the electrical charge (i.e., TD).

Another consummatory measure involved only the time during which the animal was stimulated. This procedure calculates the percentage of the time available to the animal for self-stimulation. In the FR procedures, this available time was taken into account in determining the required rate of barpressing. The train duration time and the barpress reaction time (approximately $500 \mathrm{msec}$ ) were summed and then multiplied, in the CRF and FR procedures, by the obtained number of delivered stimulations, in the SR procedure, by the frequency of selected train duration, and, in the OFF procedure, by the number representing the rewarding effect. All results are expressed in percentages of the maximal time (5 min) during which animals could self-stimulate (TS).

Choice method (CH). The apparatus used was a wooden box (60 cm long, $30 \mathrm{~cm}$ wide, and $12.5 \mathrm{~cm}$ deep) with a lever in each far wall.

The subjects were trained to self-stimulate by pressing one of the two levers. Each of these levers activated a different electrical combination to produce brain stimulation, and the rat was free to use either one. In the beginning, the animals shuttled from one lever to the other to sample the electrical combinations. The combination was held constant for $1 \mathrm{~min}$ at each lever; then the experimenter changed the two electrical combinations and a new choice was made by the animal. The total number of paired presentations of the 38 electrical combinations was 703 pairs. The percentage of choice for each electrical combination was calculated. Thus, each stimulus was paired with each of the other 37 stimulations during the choice sessions. The mean percentage of the 37 choices is given in Table 2 .

Cost method (V). The apparatus was the same as that used in the $\mathrm{CH}$ procedure but with a grid floor added. A footshock could be delivered by the experimenter to one half of the box.

The procedure tested the maximal voltage of footshock tolerated by the rat during self-stimulation sessions of $5 \mathrm{~min}$ each. The voltage was increased every $6 \mathrm{sec}$ by increments of $2 \mathrm{~V}$ until the subject escaped to the safe half of the box. Then the next electrical combination was presented to the rat, and the footshock that abolished the self-stimulation behavior was recorded (Table 2).

Weight method (W). The apparatus was the same as that employed in the CRF procedures. The lever could be loaded by the experimenter with weights of $30 \mathrm{~g}$ (=1 unit). From $30 \mathrm{~g}$ (weight of lever alone) to $300 \mathrm{~g}, 10$ units were used to render self-stimulation behavior more difficult.

Rats exhibiting steady self-stimulation behavior were able to self-stimulate for an electrical reward combination during a 1-min warm-up. Every $30 \mathrm{sec}$ thereafter, the experimenter loaded the bar with 1 unit. The session was stopped when the subject pressed the loaded lever fewer than two times for a given unit. Then the experimenter modified the electrical combination according to the same experimental procedure used in CRF. The animal selfstimulated for $1 \mathrm{~min}$ and then the experimenter again loaded the bar. The total number of leverpresses after administering the maximal unit accepted by the subject was recorded (Table 2). This estimation (WE) allowed the experimenter to measure the rate of the rat's operant response as a function of the weight loaded.

Extinction procedure (EXT). The apparatus was the same as that used in the CRF procedure.

Subjects self-stimulated for $1 \mathrm{~min}$. Then the experimenter turned off the stimulator and recorded the subjects' performance during the following minute. The criterion of extinction was $20 \mathrm{sec}$ without response. This procedure was used five times for each of the 38 electrical combinations. The duration of extinction multiplied by the number of responses during the first minute without stimulation was calculated and expressed as a time extinction (TE) stimulation.

\section{Effect of Strength of Reward on Motivational States}

Unlike the procedures in which the animals themselves regulated the rate of brain stimulation, in this phase of the study, the brain stimulation was delivered automatically, at the same rate as in the CRF procedure. This was done by recording, on magnetic tape, the frequency of barpressings for each of the 38 electrical stimuli (data presented in Table 2) and by starting the stimulator at the frequency recorded during each experimental procedure. Three different motivational states were studied: feeding, sexual, and aggressive behavior. In all three motivational states, the most typical components were selected: the frequency of feeding, genital grooming by the male rat, and aggressive dominance. All animals used had presented homogeneous rates of self-stimulation behavior.

Frequency of feeding behavior (DF). The ad-libitum rat was placed into a $30 \times 20 \times 40 \mathrm{~cm}$ Plexiglas box where its feeding behavior could be observed. The box contained a small dish of food pellets and a bottle of water.

A group of six rats was used to estimate the frequency of feeding behavior. Each rat was stimulated four times during 5-min sessions by each of the 38 electrical brain stimuli. Each session was divided into periods of $15 \mathrm{sec}$, that is, 20 periods per session. When the rat ate during one period, it was noted as one response; that is, a maximum of 20 responses per session formed a maximum of 80 responses for the four presentations per rat. It should be noted that the animal could gnaw at the pellets; no distinction was made between this behavior and a feeding behavior. Table 2 presents the mean feeding frequency of each group.

Genital grooming behavior (SZ). To observe genital self-grooming behavior, one ad-lib male rat was placed into a $60 \times 60 \times 55 \mathrm{~cm}$ box with a female rat manifesting clear lordotic behavior, that is, the behavioral manifestation of estrus. The number of copulations was too low for us to infer any relationship with the rewarding effect of brain stimuli.

Four rats were used. Each rat was observed three times during each 10-min brain stimulation session. Each session was divided into periods of $3 \mathrm{sec}$. The number of periods during which genital grooming was observed was recorded. The sum of these periods, for the three presentations of the female rat, was computed for each subject. Table 2 presents the mean sum of the sample for the 38 electrical brain stimulations.

Aggressive dominance behavior (AD). The apparatus was the box used for the genital grooming observations.

The observed rat was placed into the box 1 week before the introduction of a male partner. This procedure allowed us to establish the stimulated animal as the dominant rat and the partner as the submissive animal. Using this procedure, we hoped to show that the rewarding brain stimuli could modulate dominance behavior.

A sample of four rats was formed. The observation procedure was similar to that used for genital grooming. The frequency of aggressive dominance behavior, defined by Grant and Mackintosh (1963) as holding the partner in the submissive posture, was estimated and recorded. Table 2 shows the mean sum of the sample for 38 electrical stimuli.

\section{RESULTS}

Table 2 shows data representing the strength of the rewarding effects elicited by the 38 electrical combina- 
tions. This table shows the brain stimulation received in continuous reinforcement frequency (CRF) and in $3,5,10$, and 15 fixed ratio schedules (FR3, FR5, FR10, and FR15), the rate of train duration at which the animal self-stimulated (SR), the behavioral effects of stimuli in the switch-off procedure (OFF), the amount of electrical charge rats self-administered (CRFM, FR3M, FR5M, FR10M, FR15M, SRM, and OFFM), the percentage of time subjects were stimulated (CRFT, FR3T, FR5T, FR10T, FR15T, SRT, and OFFT), the voltage of aversive footshock required to suppress self-stimulation behavior (V), an estimation of effort on a loaded bar (W), the percentage of choice in paired electrical combination presentations $(\mathrm{CH})$, and an estimation of extinction after a self-stimulation session (EXT). The effects of reinforcing stimulations on three motivational states are also presented in Table 2-the frequency of feeding (DF), genital grooming (SZ), and aggressive dominance behavior (AD).

The problem is this: Given the many behavioral methods for measuring positive reinforcement and the possibility of altering the physical parameters of the electrical stimulus, how can we arrive at an objective measure of reinforcement that cuts across the behavioral procedures and the physical values of the stimulus?

Our aim is to compare the different procedures in order to determine whether or not the effect elicited by a given electrical combination induces the same rewarding strength. A first step is to present the data according to the stimulus duration. Figure 1 shows the shape of the curves of the strength of reward obtained in the different procedures. One could assume that, if all the curves had the same shape, the various procedures measured the strength of the electrical reward in the same manner. We observe, however, that the obtained curves differ depending on the method used. Indeed, three groups of functions seem to be present: (1) the methods in which the rat's response rate increases with the stimulus duration (CRFM, FR3M, FR5M, FR5T, FR10M, FR10T, FR15M, FR15T, OFFM, and DF); (2) the methods that result in an increasing-decreasing curve (CRF, V, FR3, FR5, FR15, W, SR, SRM, and SRT); and (3) the methods that result in an increasing-asymptotic curve ( $\mathrm{CH}, \mathrm{EXT}, \mathrm{CRFT}, \mathrm{FR3T}$, and FR10T). Moreover, as Figure 1 shows, the rat's responses in the OFF, OFFT, SZ, and AD procedures seem to be independent of brain stimulation characteristics.

These descriptive differences may be partially explained by the stimulus duration. For example, given the fixed duration of sessions of self-stimulation and the fixed duration of the electrical stimulus, the CRF procedure did not permit the rat to increase his rate of performance for long train duration. For these long TD, it appears thus logical that the amount of reward increases while the number of barpresses decreases. Nevertheless, no shape differences were found between methods in which barpressing was a dependent variable (FR3) and others in which the barpressing did not come into play $(\mathrm{V})$.

In view of the failure of this descriptive approach to explain such behavior, factor analysis has been elaborated to objectively determine the amount of reward elicited by the brain stimulation.

\section{Factor Analysis}

Bravais-Pearson correlations between methods were calculated (Snedecor \& Cochran, 1967). For each method, we have ranked the 38 electrical combinations according to their rewarding effect. A factor analysis provides five first-order factors (Figure 2).

These results seem to show that the electrical reward effect is a complex process composed of several factors. It appears, therefore, that the structure of the rewarding effect of an electrical stimulation of the hypothalamus is multifactorial. Nevertheless, the obtained factors were correlated. In other words, a hierarchical structure could be assumed. Factor analysis was performed until no further correlations between factors were obtained. Figure 2 represents the obtained hierarchical structure using the 28 procedures that estimated the rewarding effects of brain stimuli.

\section{DISCUSSION}

An electrical rewarding brain stimulation does not possess the same properties as conventional reward (Trowill, Panksepp, \& Gandelman, 1969). Nevertheless, even if self-stimulation behavior does not satisfy a biological need per se, it seems that intracranial reward has large influences on motivational states (Beyra, 1976; Hoebel, 1969; Huston, 1972; Olds, 1962; Valenstein, 1971). A common experimental procedure is to group together both the rewarding effect and the drive state induced by stimulation of a particular brain area. Establishing a reward scale to compare elicited drive states, however, is much more difficult.

According to Valenstein (1964), four classes of procedures to estimate the rewarding effect elicited by brain stimulation can be used: barpressing rate, choice procedures, costs methods, and regulation of the stimulus duration. At various stages of our investigation, we have found several other categories of methods that differ from each other, depending on the procedure used to describe the clusters (shape of curves or factor analysis). Nevertheless, Valenstein's classification is useful in comparing the curve shapes of the procedures (see the first approach).

This result stresses the assumption that the pro- 



Figure 1. Graphical presentation of the strength of the rewardig effect elicited by hypothalamic stimulation. On the abscissa, the train duration (TD) in seconds of the stimulus is presented. The pulse duration (PD), the frequency (F) of pulses, and the intensity of current remain constant in all the graphs. On the ordinate are represented the received brain stimulations per 5-min session $(\mathrm{St} / 5 \mathrm{~min})$ in the continuous reinforcement schedule (CRF) and fixed-ratio schedules (FR3-5-10-15), the rate of barpressing during self-regulation of the hypothalamic volley (SR), the complementary value of the number of switch-off behaviors (OFF), the number of microcoulombs per 5-min session $(\mu \mathrm{C} / 5 \mathrm{~min})$ consumed in CRF, SR, and OFF procedures (CRFM, FR3-5-10-15M, SRM, and OFFM), the percentage of time $(\%)$ the brain was stimulated in CRF, FR, SR, and OFF methods (CRFT, FR3-5-10-15T, SRT, OFFT), the percentage of choice (\%) of a brain stimulus paired comparison (CH), the electrical voitage (V) the arimal tolerated during self-stimulation sessions (V), the weight (W) lifted to obtain electrical stimulation of the brain, the time of extinction of selfstimulation behavior (EXT), the rate of motivated responses in aggressive (AD), feeding (DF), and sexual situations (SZ). 




Figure 2. Representation of the factor analysis computed from Table 2. The hierarchy of factors in first- and second-order approximation is established by means of correlations between the 28 variables. The position of each method is determined by the highest saturation in a factor.

cedures used are quite alike in their capacity to estimate the rewarding effect induced by brain stimulations.

On the basis of these data, it appears difficult to reject methods on a unilateral basis. Bitterman and Schoel (1970) stressed five parameters in estimating the reinforcing effect elicited by rewarding stimuli. They distinguished the number, contrast, delay, frequency, and probability of reward events. Our paper deals with the first parameters (i.e., the amount of reward) by assuming that the more the behavior produces a rewarding brain stimulation, the stronger the rewarding effect.

Regarding the factor analysis that specifies clustering of the rewarding effect of intracranial stimulation (Figure 2), we assume that the methods saturated by the same factor measure the same rewarding effect. It seems that Factor 1 includes consummatory methods (CRFM, FR3M, FR5M, FR10M, FR15M, FR3T, FR5T, FR10T, FR15T, SRM, and OFFM), Factor 2 includes methods requiring discrimination between brain stimuli on the basis of the rewarding quality (i.e., the rats have to modulate their behavior and execute an operant procedure before obtaining the reward) (FR3, FR5, FR10, FR15, W, EXT, and OFF), and Factor 4 includes methods requiring discrimination on the basis of the quantity of reward they induce (i.e., the rats have to work more intensively to obtain the stronger rewarding effect) (CRF). Factor 3, including the frequency of eating behavior (DF) and regulation of duration of brain stimuli (SR), clusters together the consummatory methods in Factor 1' of second-order factor analysis. The two sets of discriminatory methods are grouped together in Factor 2' of second-order analysis. Finally, a thirdorder factor analysis assembles into one factor all the methods measuring the rewarding effect, that is, the global effect of brain stimuli. Furthermore, it seems that the "motivational methods" were either positively related to other methods (DF) or negatively related (SZ in Factor 1 and AD show negative values between Factor $3^{\prime}$ and Factor $1^{\prime \prime}$ ). These relationships, disregarding the neural system that is effectively excited, suggest that the drive state could either induce or reduce reinforcing strength of hypothalamic stimulation. In other words, sexual or aggressive behavior seems to reduce the reinforcing effect of brain stimulation, while eating behavior seems to increase it. Thus, when the satisfaction of a drive requires the presence of a congener (male or female), this satisfaction reduces the intracranial reinforcing effect. However, when the satisfaction of a drive requires no congener, such as during eating, then the satiation of the drive state could induce a reinforcing effect.

Apart from these mathematical relationships, the theoretical interpretation of the relations between the reinforcing effect and the drive state elicited by hypothalamic stimulation remains difficult. Assuming that brain stimulation does not elicit a specific state but a general "mood state" (Valenstein, 1969), it appears that the third-order factor, that is, the global rewarding state, could be the mathematical manifestation of this general "mood state." On the other hand, Hoebel's (1971) drive induction hypothesis seems to correspond to the $\mathbf{1}^{\prime}$ factor of the second-order factor analysis. In fact, the greater the augmentation of the drive effect, the stronger the reinforcing effect of hypothalamic stimulation and the more the animal eats. Finally, a third hypothesis is Ball's drive reduction hypothesis (Ball, 1969; Huston, 1971), which postulates that a decrease in the drive state elicited by brain stimulation is accompanied by an increase in reinforcing strength, and which seems to apply to the sexual and aggressive components studied. These controversies between hypotheses show how complex the nature of the relationship between reinforcing and drive effects elicited by hypothalamic stimulation is. Furthermore, these data suggest that the interpretation of the relationship between self-stimulation behavior and goaloriented behavior depends, first, on the ability to interpret the multifactorial nature of the reinforcing effect, and second, on the structure of the goaldirected behavior studied. It seems that this structure depends on the neural system excited by the electrode as well as the social structure of the behavior, indicating environmental influences on self-stimulation and drive state. 


\section{REFERENCES}

BALL, G. C. Separation of electrical self-stimulation and electrically elicited eating in the hypothalamus. Communications in Behavioral Biology, 1969, 17, 5-10.

BEYRA, M. The measurement of reinforcing brain stimulation. Brain Research, 1974, 70, 467-479.

BEYRA, M. Relations between stimulus-bound eating and intracranial reinforcing strength. Behavioral Processes, 1976, 1, 165-175.

Bitterman, M. E., \& Schoel, M. W. Instrumental learning in animals: Parameters of reinforcement. Annual Review of Psychology, 1970, 21, 367-436.

Brodie, D., Moreno, O. M., Malis, J. M., \& Boren, J. J. Nonreversibility of the appetitive characteristics of intracranial stimulation. American Journal of Physiology, 1960, 199, 707-709.

DE WitTe, PH. Reinforcing stimulation of the postero-lateral hypothalamus and mating behavior in the rat. Archives Internationales de Physiologie et Biochimie, 1978, 86, 985-995.

Gallistel, C. R. Electrical self-stimulation and its theoretical implications. Psychological Bulletin, 1964, 61, 23-24.

Grant, E. C., \& Mackintosh, J. H. A comparison of the social posture of some common laboratory rodents. Behaviour, 1963, 21, 246-259.

Hodos, W., \& Valenstein, E. S. An evaluation of response rate as a measure of rewarding intracranial stimulation. Journal of Comparative and Physiological Psychology, 1962, 55, 80-84.

Hoebel, B. G. Feeding and self-stimulation. Annals of the New York Academy of Sciences, 1969, 157, 758-778.

HoEbel, B. G. Feeding: Neural control of intake. Annual Review of Physiology, 1971, 33, 533-568.

Huston, J. P. Relationship between motivating and rewarding stimulation of the lateral hypothalamus. Physiology \& Behavior, 1971, 6, 711-716.

Huston, J. P. Inhibition of hypothalamically motivated eating by rewarding stimulation through the same electrode. Physiology \& Behavior, 1972, 8, 1121-1125.

KEESEY, R. E. Duration of stimulation and the reward properties of hypothalamic stimulation. Journal of Comparative and Physiological Psychology, 1964, 58, 201-207.

Ouds, J. Hypothalamic substrate of reward. Physiological Review, $1962,42,554-604$.

Olds, J., \& MilnER, P. Positive reinforcement produced by electrical stimulation of septal area and other regions of the brain. Journal of Comparative and Physiological Psychology, 1954, 47, 419-427.

Olds, M. E., \& OLDS, J. Approach-avoidance analysis of rat diencephalon. Journal of Comparative Neurology, 1963, 120 , 259-295.

Sidman, M., Brady, J. V., Conrad, D. G., \& Schulman, A. Reward schedules and behavior maintained by intracranial selfstimulation. Science, 1955, 122, 830-831.

Snedecor, G. W., \& Cochran, W. G. Statistical methods (6th ed.). Ames: Iowa State University Press, 1967.

Trowill, J. A., Pankse PP, J., \& Gandelman, R. An incentive model of rewarding brain stimulation. Psychological Review, 1969, 76, 264-281.

VAlenstein, E. S. Problems of measurement and interpretation with reinforcing brain stimulation. Psychological Review, 1964, 71, 415-437.

VAlenstein, E. S. Behavior elicited by hypothalamic stimulation. Brain and Behavior Evolution, 1969, 2, 295-316.

VALENSTE IN, E. S. Channeling of responses elicited by hypothalamic stimulation. Journal of Psychiatric Research, 1971, 8 , 335-344.

WhITE, N. Effects of anterior medial forebrain bundle lesions on self-stimulation with two different operant responses. Behavioral Biology, 1975, 14, 575-591.

(Received for publication April 12, 1979; revision accepted February 2, 1980.) 